\title{
Deformation Argument under PSP Condition and Applications
}

\author{
Silvia Cingolani ${ }^{1, *}$ and Kazunaga Tanaka ${ }^{2}$ \\ ${ }^{1}$ Dipartimento di Matematica, Università degli Studi di Bari Aldo Moro, Via E. \\ Orabona 4, 70125 Bari, Italy \\ ${ }^{2}$ Department of Mathematics, School of Science and Engineering, Waseda \\ University, 3-4-1 Ohkubo, Shijuku-ku, Tokyo 169-8555, Japan
}

Received 14 August 2020; Accepted (in revised version) 28 October 2020

Dedicated to Prof. Paul H. Rabinowitz with admiration on the occasion of his 80th birthday

\begin{abstract}
In this paper we introduce a new deformation argument, in which $C^{0}$-group action and a new type of Palais-Smale condition PSP play important roles. This type of deformation results are studied in $[17,21]$ and has many different applications $[10$, $11,17,21]$ et al. Typically it can be applied to nonlinear scalar field equations. We give a survey in an abstract functional setting. We also present another application to nonlinear elliptic problems in strip-like domains. Under conditions related to [5,6], we show the existence of infinitely many solutions. This extends the results in [8].
\end{abstract}

Key Words: Deformation theory, nonlinear elliptic equations, radially symmetric solutions, striplike domains, Pohozaev functional.

AMS Subject Classifications: 35B38, 35J20, 58E05

\section{Introduction}

We study nonlinear differential equations with scaling properties via variational methods. A typical example is the following nonlinear scalar field equations:

$$
\begin{aligned}
-\Delta u & =g(u) \quad \text { in } \mathbf{R}^{N}, \\
u & \in H^{1}\left(\mathbf{R}^{N}\right),
\end{aligned}
$$

where $N \geq 2$ and we consider the existence of radially symmetric solutions. This type of problem appears in many models in mathematical physics and is well-studied by many authors. Especially Berestycki and Lions [5,6] and Berestycki, Gallouët and Kavian [7] obtained almost necessary and sufficient conditions for the existence of non-trivial solutions. More precisely they consider (1.1) under the following conditions

*Corresponding author. Email addresses: kazunaga@waseda.jp (K. Tanaka), silvia.cingolani@uniba.it (S. Cingolani) 
(g0) $g(\xi) \in C(\mathbf{R}, \mathbf{R})$ and $g(\xi)$ is odd.

(g1) For $N \geq 3$,

$$
\limsup _{\xi \rightarrow \infty} \frac{g(\xi)}{\xi^{(N+2) /(N-2)}} \leq 0
$$

For $N=2$,

$$
\limsup _{\xi \rightarrow \infty} \frac{g(\xi)}{e^{\alpha \xi^{2}}} \leq 0 \quad \text { for any } \quad \alpha>0
$$

(g2) $-\infty<\liminf _{\xi \rightarrow 0} \frac{g(\xi)}{\xi} \leq \limsup _{\xi \rightarrow 0} \frac{g(\xi)}{\xi}<0$.

(g3) There exists a $\zeta_{0}>0$ such that $G\left(\zeta_{0}\right)>0$, where $G(\xi)=\int_{0}^{\xi} g(\tau) d \tau$.

The [5,6] (for $N \geq 3$ ) and [7] (for $N=2$ ) showed the existence of a positive solution of (1.1) and infinitely many possibly sign-changing radially symmetric solutions. We note that in [5-7] solutions are found as critical points of constraint functional

$$
\begin{array}{ll}
u \mapsto \int_{\mathbf{R}^{N}}|\nabla u|^{2} d x ;\left\{u \in H_{r}^{1}\left(\mathbf{R}^{N}\right) ; \int_{\mathbf{R}^{N}} G(u) d x=1\right\} \rightarrow \mathbf{R} & \text { for } N \geq 3, \\
u \mapsto \int_{\mathbf{R}^{2}}|\nabla u|^{2} d x ;\left\{u \in H_{r}^{1}\left(\mathbf{R}^{2}\right) ; \int_{\mathbf{R}^{2}} G(u) d x=0\right\} \rightarrow \mathbf{R} & \text { for } N=2,
\end{array}
$$

after a suitable scaling and solutions satisfy Pohozaev identity. See Coleman, Glazer and Martin [12] for related argument. We also note that a positive solution is obtained as a minimizer after scaling and it is a least energy solution.

Remark 1.1. When $N=2$, in [7] the existence of solution is obtained under slightly stronger conditions (g0), (g1), (g3) and

(g2') $\lim _{\xi \rightarrow 0} \frac{g(\xi)}{\xi}<0$ exists.

In [16], Hirata, Ikoma and the second author introduced a new approach to (1.1), in which we try to apply minimax argument to the natural functional associated to (1.1):

$$
I(u)=\frac{1}{2} \int_{\mathbf{R}^{N}}|\nabla u|^{2} d x-\int_{\mathbf{R}^{N}} G(u) d x: H_{r}^{1}\left(\mathbf{R}^{N}\right) \rightarrow \mathbf{R} .
$$

We note that it is difficult to verify so-called Palais-Smale condition $((P S)$ in short) for $I(u)$ and we cannot apply the standard deformation argument directly to $I(u)$. We also remark that the constraint functional (1.2a) and (1.2b) satisfy $(P S)$ condition.

To avoid lack of (PS) condition, we make use of a special scaling property of the functional $I(u)$ and we introduce following Pohozaev functional:

$$
P(u)=\frac{N-2}{2} \int_{\mathbf{R}^{N}}|\nabla u|^{2} d x-N \int_{\mathbf{R}^{N}} G(u) d x: H_{r}^{1}\left(\mathbf{R}^{N}\right) \rightarrow \mathbf{R} .
$$


Using the scaling $u_{\lambda}(x)=u(x / \lambda)$, we have formally

$$
\begin{aligned}
P(u) & =-I^{\prime}(u)(x \cdot \nabla u)=I^{\prime}(u)\left(\left.\frac{d}{d \lambda}\right|_{\lambda=1} u_{\lambda}\right) \\
& =\left.\frac{d}{d \lambda}\right|_{\lambda=1} I\left(u_{\lambda}\right) .
\end{aligned}
$$

In [16], Hirata, Ikoma and the second author found a $(P S)$ sequence with an extra property at minimax level $b=\inf _{\gamma \in \Gamma} \max _{\xi \in D} I(\gamma(\xi))$. That is, there exists a sequence $\left(u_{j}\right)_{j=1}^{\infty} \subset$ $H_{r}^{1}\left(\mathbf{R}^{N}\right)$ such that as $j \rightarrow \infty$

$$
\begin{aligned}
& I\left(u_{j}\right) \rightarrow b, \\
& I^{\prime}\left(u_{j}\right) \rightarrow 0 \quad \text { in }\left(H_{r}^{1}\left(\mathbf{R}^{N}\right)\right)^{*}, \\
& P\left(u_{j}\right) \rightarrow 0 .
\end{aligned}
$$

For example, at a mountain pass level for $I(u)$, they find a sequence $\left(u_{j}\right)_{j=1}^{\infty}$ with (1.4a)(1.4c) and under the condition (g0)-(g3) and moreover they show that $\left(u_{j}\right)_{j=1}^{\infty}$ has a strongly convergent subsequence whose limit is a solution of (1.1). See Remark 4.1 in Section 4 .

The condition (1.4c) means $\left(u_{j}\right)_{j=1}^{\infty}$ satisfies Pohozaev identity $P(u)=0$ asymptotically and we call such sequence $\left(u_{j}\right)_{j=1}^{\infty}$ as $(P S P)$ sequences.

Existence of such (PSP) sequences was firstly found by the second author. Jeanjean [22] used the second author's approach for $L^{2}$ normalized solutions for $L^{2}$ super critical problems and in [16], we studied nonlinear scalar field equation (1.1) through (PSP) sequence and showed the existence of positive radially symmetric solutions via mountain pass method.

Such a strategy turned out to be useful for various problems with suitable scaling properties. See [23] for an application for nonlinear Choquard equations, [2,18-20] for fractional scalar field equations, [9] for FitzHugh-Nagumo elliptic systems, [8] for nonlinear elliptic equations in strip-like domains, [3] for nonlinear Schrödiger-Maxwell systems, [4] for nonlinear eigenvalue problems.

For even functionals, it is natural to ask the existence of infinitely many solutions. We note that our argument in [16] does not provide a deformation theory for $I(u)$ and we cannot apply genus theory directly to $I(u)$. So to find infinitely many solutions, we need to use some comparison argument to ensure the existence of unbounded sequence of minimax values. See $[2,9,16]$.

In this paper we give a survey of some deformation theorems contained in previous papers $[10,11,17,21]$ and a new application to semilinear elliptic equations in strip-like domains. Our deformation result works for $I(u)$ under $(\mathrm{g} 0)-(\mathrm{g} 3)$ and enables us to apply genus theory directly to $I(u)$. It also shows that critical points with Pohozaev identity are essential in the deformation argument (see Corollary 3.1 and Remark 3.1 in Section 3). A special scaling property and a new type of Palais-Smale condition, which we call (PSP) 
condition and which claims any (PSP) sequence has a strongly convergent subsequence, play important roles in our argument. We give our deformation result in a general setting in Sections 2-3. We also give an existence result for $(P S P)$ sequence at a minimax level in Section 4.

We note that such a deformation argument is firstly given in [17] for $L^{2}$ normalized problem for nonlinear scalar field equations. We also refer to [21] for $L^{2}$ super critical problems and $[10,11]$ for our recent works on $L^{2}$ normalized solutions for nonlinear Choquard equations and fractional nonlinear scalar field equations.

In Section 5, we give a new application to a nonlinear elliptic problem in a strip-like domain:

$$
\begin{aligned}
& -\Delta u=f(u) \text { in } \mathbf{R}^{k} \times D, \\
& u \in H_{0}^{1}\left(\mathbf{R}^{k} \times D\right),
\end{aligned}
$$

where $k \geq 2$. Under conditions (f0)-(f3) in Section 5 , which are related to (g0)-(g3), we show the existence of infinitely many solutions.

\section{Deformation argument under $(P S P)$}

We give our deformation argument in an abstract framework. Let $\left(E,\|\cdot\|_{E}\right)$ be a Hilbert space and $\Phi: \mathbf{R} \rightarrow L(E) ; \theta \mapsto \Phi_{\theta}$ be a continuous group action of $\mathbf{R}$. For $I \in C^{1}(E, \mathbf{R})$ we assume

\section{Assumption 2.1.}

(i) $\Phi_{\theta}$ is a $C^{0}$-group action, that is,

$$
\begin{aligned}
& \Phi_{0}=i d, \\
& \Phi_{\theta+\theta^{\prime}}=\Phi_{\theta} \circ \Phi_{\theta^{\prime}} \text { for all } \theta, \theta^{\prime} \in \mathbf{R}, \\
& \theta \mapsto \Phi_{\theta} u ; \mathbf{R} \rightarrow E \text { is continuous for all } u \in E .
\end{aligned}
$$

(ii) Let $M=\mathbf{R} \times E$ and we regard $M$ as a Hilbert manifold and we introduce a metric by

$$
\|(\kappa, v)\|_{(\theta, u)}=\left(|\kappa|^{2}+\left\|\Phi_{\theta} u\right\|_{E}^{2}\right)^{1 / 2}
$$

for all $(\kappa, v) \in \mathbf{R} \times E=T_{(\theta, u)} M$ and $(\theta, u) \in M$. We assume $\|\cdot\|_{(\theta, u)}$ is a metric of class $C^{2}$.

(iii) Let

$$
J(\theta, u)=I\left(\Phi_{\theta} u\right): M \rightarrow \mathbf{R} .
$$

Then we assume that $J(\theta, u) \in C^{1}(M, \mathbf{R})$. 
Under the Assumption 2.1, we introduce

$$
P(u)=\frac{\partial J}{\partial \theta}(0, u): E \rightarrow \mathbf{R} .
$$

For $b \in \mathbf{R}$ we request the following Palais-Smale type condition $(P S P)_{b}$ for $I(u)$. $(P S P)_{b}$ Assume that $\left(u_{j}\right)_{j=1}^{\infty} \subset E$ satisfies

$$
\begin{aligned}
& I\left(u_{j}\right) \rightarrow b, \\
& I^{\prime}\left(u_{j}\right) \rightarrow 0 \text { in } E^{*}, \\
& P\left(u_{j}\right) \rightarrow 0 .
\end{aligned}
$$

Then $\left(u_{j}\right)_{j=1}^{\infty}$ has a strongly convergent subsequence.

Under the above assumptions, we have the following deformation result in which we use notation:

$$
\begin{aligned}
& K_{b}=\left\{u \in E ; I(u)=b, I^{\prime}(u)=0, P(u)=0\right\} \\
& {[I \leq c]=\{u \in E ; I(u) \leq c\} \text { for } c \in \mathbf{R} .}
\end{aligned}
$$

We note that $K_{b}$ is different from the usual critical set at level $b$ and it requests $P(u)=0$.

Theorem 2.1. Assumption 2.1 and for $b \in \mathbf{R}(P S P)_{b}$ holds. Then

(i) $K_{b}$ is compact in $E$.

(ii) For any neighborhood $O$ of $K_{b}$ and for any $\bar{\varepsilon}>0$ there exists $\varepsilon \in(0, \bar{\varepsilon})$ and a continuous map $\eta(t, u):[0,1] \times E \rightarrow$ E such that

(1) $\eta(0, u)=u$ for all $u \in E$.

(2) $\eta(t, u)=u$ for all $t \in[0,1]$ and $u \in[I \leq b-\bar{\varepsilon}]$.

(3) $I(\eta(t, u)) \leq I(u)$ for all $(t, u) \in \mathbf{R} \times E$.

(4) $\eta(1,[I \leq b+\varepsilon] \backslash O) \subset[I \leq b-\varepsilon], \eta(1,[I \leq b+\varepsilon]) \subset[I \leq b-\varepsilon] \cup O$.

(5) If $K_{b}=\varnothing$, then $\eta(1,[I \leq b+\varepsilon]) \subset[I \leq b-\varepsilon]$.

(6) If $I(u)$ is an even functional, then $\eta(t,-u)=-\eta(t, u)$ for all $(t, u) \in \mathbf{R} \times E$.

A typical situation, where the Assumption 2.1 is satisfied, is given in the following example.

Example 2.1. Let $E=H_{r}^{1}\left(\mathbf{R}^{N}\right)$ and

$$
\|u\|_{E}=\left(\int_{\mathbf{R}^{N}}|\nabla u|^{2}+|u|^{2} d x\right)^{1 / 2} .
$$


We consider the following action $\Phi: \mathbf{R} \rightarrow L\left(H_{r}^{1}\left(\mathbf{R}^{N}\right)\right)$ defined by

$$
\left(\Phi_{\theta} u\right)(x)=u\left(x / e^{\theta}\right) .
$$

Then we have

$$
\begin{aligned}
& \|(\kappa, v)\|_{(\theta, u)}^{2}=|\kappa|^{2}+\left\|u\left(x / e^{\theta}\right)\right\|_{H^{1}}^{2} \\
= & |\kappa|^{2}+e^{(N-2) \theta}\|\nabla u\|_{L^{2}}^{2}+e^{N \theta}\|u\|_{L^{2}}^{2} .
\end{aligned}
$$

For $I(u) \in C^{1}(E, \mathbf{R})$ given in (1.3), we have

$$
\begin{aligned}
& J(\theta, u)=I\left(u\left(x / e^{\theta}\right)\right) \\
= & \frac{1}{2} e^{(N-2) \theta}\|\nabla u\|_{L^{2}}^{2}-e^{N \theta} \int_{\mathbf{R}^{N}} G(u) d x \in C^{1}(\mathbf{R} \times E, \mathbf{R}) .
\end{aligned}
$$

We note that $\theta \mapsto \Phi_{\theta} u$ is not of class $C^{1}$ in general but $J(\theta, u)$ is of class $C^{1}$. That is, $E, \Phi_{\theta}$ satisfy the Assumption 2.1. We also note that

$$
P(u)=\partial_{\theta} J(0, u)=\frac{N-2}{2} \int_{\mathbf{R}^{N}}|\nabla u|^{2} d x-N \int_{\mathbf{R}^{N}} G(u) d x .
$$

We also note that under $(\mathrm{g} 0)-(\mathrm{g} 3), I(u)$ satisfies $(P S P)_{b}$ condition for all $b \in \mathbf{R}$ (see Proposition 6.1 in [17]) and we can find infinitely many solutions via symmetric mountain pass theorem.

The statement (i) of Theorem 2.1 is a direct consequence from $(P S P)_{b}$. In Section 3, we give an outline of the proof of the statement (ii).

Remark 2.1. Theorem 2.1 can be generalized to the setting, where a functional $I(u)$ is defined on a submanifold in $E$. See [21], where applications to $L^{2}$ normalized solutions are also given.

\section{Outline of the proof of Theorem 2.1}

Proof of Theorem 2.1 is given using a deformation flow for $J(\theta, u): M \rightarrow \mathbf{R}$. We begin with some notation. First we define the standard distance $\operatorname{dist}_{M}$ on $M$ by

$$
\begin{aligned}
& \operatorname{dist}_{M}\left(\left(\theta_{0}, u_{0}\right),\left(\theta_{1}, u_{1}\right)\right) \\
= & \inf \left\{\int_{0}^{1}\left\|\frac{d \sigma}{d t}(t)\right\|_{\sigma(t)} d t ; \sigma(t) \in C^{1}([0,1], M), \sigma(i)=\left(\theta_{i}, u_{i}\right) \text { for } i=0,1\right\} .
\end{aligned}
$$

We have easily that

$$
\operatorname{dist}_{M}\left(\left(\theta_{0}+\alpha, u_{0}\right),\left(\theta_{1}+\alpha, u_{1}\right)\right)=\operatorname{dist}_{M}\left(\left(\theta_{0}, \Phi_{\alpha} u_{0}\right),\left(\theta_{1}, \Phi_{\alpha} u_{1}\right)\right) .
$$


For $F \in T_{(\theta, u)}^{*} M$, we define its norm by

$$
\|F\|_{(\theta, u), *}=\sup \left\{F(\kappa, v) ;(\kappa, v) \in \mathbf{R} \times E,\|(\kappa, v)\|_{(\theta, u)} \leq 1\right\} .
$$

Writing $D=\left(\partial_{\theta}, \partial_{u}\right)$, we have

$$
D J(\theta, u)(\kappa, v)=\partial_{\theta} J(\theta, u) \kappa+\partial_{u} J(\theta, u) v .
$$

By the definition of $J(\theta, u)$, we have for $(\theta, u) \in M$

$$
\begin{aligned}
& J(\theta, u)=J\left(0, \Phi_{\theta} u\right)=I\left(\Phi_{\theta} u\right), \\
& J(\theta+\alpha, u)=J\left(\alpha, \Phi_{\theta} u\right) \text { for } \alpha \in \mathbf{R}, \\
& \partial_{\theta} J(\theta, u)=\partial_{\theta} J\left(0, \Phi_{\theta} u\right)=P\left(\Phi_{\theta} u\right), \\
& \partial_{u} J(\theta, u) v=\partial_{u} J\left(0, \Phi_{\theta} u\right) \Phi_{\theta} v=I^{\prime}\left(\Phi_{\theta} u\right) \Phi_{\theta} v .
\end{aligned}
$$

In particular,

$$
\begin{aligned}
& \|D J(\theta, u)\|_{(\theta, u), *}=\left\|D J\left(0, \Phi_{\theta} u\right)\right\|_{\left(0, \Phi_{\theta} u\right), *} \\
= & \left(\left|P\left(\Phi_{\theta} u\right)\right|^{2}+\left\|I^{\prime}\left(\Phi_{\theta} u\right)\right\|_{E^{*}}^{2}\right)^{1 / 2} .
\end{aligned}
$$

Finally for $b \in \mathbf{R}$, we set

$$
\widetilde{K}_{b}=\{(\theta, u) \in M ; J(\theta, u)=b, D J(\theta, u)=0\} .
$$

We note that

$$
\begin{aligned}
& (\theta, u) \in \widetilde{K}_{b} \quad \text { if and only if } \Phi_{\theta} u \in K_{b}, \\
& \widetilde{K}_{b}=\left\{\left(\alpha, \Phi_{-\alpha} u\right) ; u \in K_{b}, \alpha \in \mathbf{R}\right\}, \\
& \operatorname{dist}_{M}\left((\theta, u), \widetilde{K}_{b}\right)=\operatorname{dist}_{M}\left(\left(0, \Phi_{\theta} u\right), \widetilde{K}_{b}\right) \leq \operatorname{dist}_{E}\left(\Phi_{\theta} u, K_{b}\right) .
\end{aligned}
$$

From the above properties we have

Lemma 3.1. Suppose Assumption 2.1 holds and for $b \in \mathbf{R}$ assume $(P S P)_{b}$. Then (i) Let $\left(\theta_{j}, u_{j}\right) \subset M$ satisfies

$$
J\left(\theta_{j}, u_{j}\right) \rightarrow b, \quad\left\|D J\left(\theta_{j}, u_{j}\right)\right\|_{\left(\theta_{j}, u_{j}\right), *} \rightarrow 0 \quad \text { as } j \rightarrow \infty .
$$

Then $\left(\Phi_{\theta_{j}} u_{j}\right)_{j=1}^{\infty}$ has a strongly convergent subsequence in $E$. Moreover we have

$$
\operatorname{dist}_{M}\left(\left(\theta_{j}, u_{j}\right), \widetilde{K}_{b}\right) \rightarrow 0 \text { as } j \rightarrow \infty .
$$

(ii) Suppose $K_{b} \neq \varnothing$, i.e., $\widetilde{K}_{b} \neq \varnothing$. Then for any $\rho>0$ there exists $\delta_{\rho}>0$ such that

$$
\|D J(\theta, u)\|_{(\theta, u), *} \geq \delta_{\rho} \quad \text { if } J(\theta, u) \in\left[b-\delta_{\rho}, b+\delta_{\rho}\right] \text { and }(\theta, u) \notin \widetilde{N}_{\rho}\left(\widetilde{K}_{b}\right) .
$$


Here

$$
\widetilde{N}_{\rho}\left(\widetilde{K}_{b}\right)=\left\{(\theta, u) ; \operatorname{dist}_{M}\left((\theta, u), \widetilde{K}_{b}\right)<\rho\right\} .
$$

(iii) If $K_{b}=\varnothing$, i.e., $\widetilde{K}_{b}=\varnothing$, there exists $\delta_{0}>0$ such that

$$
\|D J(\theta, u)\|_{(\theta, u), *} \geq \delta_{0} \quad \text { for }(\theta, u) \in M \quad \text { with } J(\theta, u) \in\left[b-\delta_{0}, b+\delta_{0}\right] .
$$

Proof. By (3.3) and $(P S P)_{b}$, (i) follows. (ii) and (iii) follow from (i) easily.

We use notation

$$
[J \leq c]_{M}=\{(\theta, u) \in M ; J(\theta, u) \leq c\} \quad \text { for } c \in \mathbf{R} .
$$

By (ii), (iii) of Lemma 3.1, for any $\rho>0$ there exists $\delta_{\rho}^{\prime}>0$ such that

$$
\|D J(\theta, u)\|_{(\theta, u), *} \geq \delta_{\rho}^{\prime}
$$

for $(\theta, u) \in\left(\left[J \leq b+\delta_{\rho}^{\prime}\right]_{M} \backslash\left[J \leq b-\delta_{\rho}^{\prime}\right]_{M}\right) \backslash \widetilde{N}_{\frac{1}{3} \rho}\left(\widetilde{K}_{b}\right)$.

Taking a pseudo-gradient vector field

$$
\mathcal{V}(\theta, u):\left(\left[J \leq b+\delta_{\rho}^{\prime}\right]_{M} \backslash\left[J \leq b-\delta_{\rho}^{\prime}\right]_{M}\right) \backslash \widetilde{N}_{\frac{1}{3} \rho}\left(\widetilde{K}_{b}\right) \rightarrow \mathbf{R} \times E
$$

corresponding to $D J$ and choosing suitable cut-off functions $\varphi, \psi: E \rightarrow[0,1]$ such that

$$
\begin{aligned}
& \varphi(\theta, u)= \begin{cases}1 & \text { for }(\theta, u) \in M \backslash \widetilde{N}_{\frac{2}{3} \rho}\left(\widetilde{K}_{b}\right), \\
0 & \text { for }(\theta, u) \in \widetilde{N}_{\frac{1}{3} \rho}\left(\widetilde{K}_{b}\right),\end{cases} \\
& \psi(\theta, u)= \begin{cases}1 & \text { if } J(\theta, u) \in\left[b-\frac{1}{2} \delta_{\rho}^{\prime}, b+\frac{1}{2} \delta_{\rho}^{\prime}\right], \\
0 & \text { if } J(\theta, u) \notin\left[b-\delta_{\rho}^{\prime}, b+\delta_{\rho}^{\prime}\right],\end{cases}
\end{aligned}
$$

we consider the following ODE in $M$ :

$$
\begin{aligned}
& \frac{d \widetilde{\eta}}{d t}=-\varphi(\widetilde{\eta}) \psi(\widetilde{\eta}) \frac{\mathcal{V}(\widetilde{\eta})}{\|\mathcal{V}(\widetilde{\eta})\|_{\widetilde{\eta}}}, \\
& \widetilde{\eta}(0, \theta, u)=(\theta, u) .
\end{aligned}
$$

In a standard way, we have the following

Proposition 3.1. For any $\bar{\varepsilon}>0$ and $\rho>0$, there exist $\varepsilon \in(0, \bar{\varepsilon})$ and $\widetilde{\eta} \in C([0,1] \times M, M)$ such that

(i) $\widetilde{\eta}(0, \theta, u)=(\theta, u)$ for $(\theta, u) \in M$.

(ii) $\widetilde{\eta}(t, \theta, u)=(\theta, u)$ for $t \in[0,1]$ if $(\theta, u) \in[J \leq b-\bar{\varepsilon}]_{M}$. 
(iii) $t \mapsto J(\widetilde{\eta}(t, \theta, u))$ is non-increasing for $(\theta, u) \in M$.

(iv) $\left.\widetilde{\eta}\left(1,[J \leq b+\varepsilon]_{M}\right) \backslash \widetilde{N}_{\rho}\left(\widetilde{K}_{b}\right)\right) \subset[J \leq b-\varepsilon]_{M}, \widetilde{\eta}\left(1,[J \leq b+\varepsilon]_{M}\right) \subset[J \leq b-\varepsilon]_{M} \cup$ $\widetilde{N}_{\rho}\left(\widetilde{K}_{b}\right)$.

(v) If $K_{b}=\varnothing$, i.e., $\widetilde{K}_{b}=\varnothing$, we have $\widetilde{\eta}\left(1,[J \leq b+\varepsilon]_{M}\right) \subset[J \leq b-\varepsilon]_{M}$.

(vi) If I $(u)$ is even in $u, \widetilde{\eta}(t, \theta, u)=\left(\widetilde{\eta}_{1}(t, \theta, u), \widetilde{\eta}_{2}(t, \theta, u)\right)$ satisfies

$$
\widetilde{\eta}_{1}(t, \theta,-u)=\widetilde{\eta}_{1}(t, \theta, u), \quad \widetilde{\eta}_{2}(t, \theta,-u)=-\widetilde{\eta}_{2}(t, \theta, u) .
$$

Our Theorem 2.1 can be derived from Proposition 3.1. We need the following operator:

$$
\pi: M=\mathbf{R} \times E \rightarrow E ;(\theta, u) \mapsto \Phi_{\theta} u .
$$

We need the following lemma.

Lemma 3.2. For any $\rho>0$ there exists a $R(\rho)>0$ such that

$$
\begin{aligned}
& \pi\left(\widetilde{N}_{\rho}\left(\widetilde{K}_{b}\right)\right) \subset N_{R(\rho)}\left(K_{b}\right), \\
& R(\rho) \rightarrow 0 \text { as } \rho \rightarrow 0 .
\end{aligned}
$$

Here

$$
N_{r}\left(K_{b}\right)=\left\{u \in E ; \operatorname{dist}_{E}\left(u, K_{b}\right)<r\right\} .
$$

Proof. Suppose that $(\theta, u) \in \widetilde{N}_{\rho}\left(\widetilde{K}_{b}\right)$. By (3.1), note that

$$
\operatorname{dist}_{M}\left(\left(0, \Phi_{\theta} u\right), \widetilde{K}_{b}\right)=\operatorname{dist}_{M}\left((\theta, u), \widetilde{K}_{b}\right)<\rho
$$

and choose a $\sigma(t) \in C^{1}([0,1], M)$ such that $\sigma(0)=\left(0, \Phi_{\theta} u\right), \sigma(1) \in \widetilde{K}_{b}$,

$$
\int_{0}^{1}\left\|\frac{d \sigma}{d t}(t)\right\|_{\sigma(t)} d t<\rho .
$$

Writing $\sigma(t)=\left(\sigma_{1}(t), \sigma_{2}(t)\right)$, we have

$$
\left|\sigma_{1}(t)\right| \leq \int_{0}^{1}\left|\frac{d \sigma_{1}}{d t}(t)\right| d t \leq \int_{0}^{1}\left\|\frac{d \sigma}{d t}(t)\right\|_{\sigma(t)} d t<\rho .
$$

We note that there exists $c_{\rho}>0$ such that for some $\delta \in(0,1]$

$$
\begin{array}{ll}
c_{\rho}\|v\|_{E} \leq\left\|\Phi_{\theta} v\right\|_{E} & \text { for }|\theta| \leq \rho \text { and } v \in E, \\
\delta \leq c_{\rho} \leq 1 & \text { for } \rho \in(0,1] .
\end{array}
$$


Thus

$$
\begin{aligned}
\operatorname{dist}_{E}\left(\Phi_{\theta} u, \sigma_{2}(1)\right) & \leq \int_{0}^{1}\left\|\frac{d \sigma_{2}}{d t}(t)\right\|_{E} d t \leq c_{\rho}^{-1} \int_{0}^{1}\left\|\Phi_{\sigma_{1}(t)} \frac{d \sigma_{2}}{d t}(t)\right\|_{E} d t \\
& \leq c_{\rho}^{-1} \int_{0}^{1}\left\|\frac{d \sigma}{d t}(t)\right\|_{\sigma(t)} d t \leq c_{\rho}^{-1} \rho .
\end{aligned}
$$

Therefore, noting $\Phi_{\sigma_{1}(1)} \sigma_{2}(1) \in K_{b}$,

$$
\begin{aligned}
\operatorname{dist}_{E}\left(\pi(\theta, u), K_{b}\right) & =\operatorname{dist}_{E}\left(\Phi_{\theta} u, K_{b}\right) \leq \operatorname{dist}_{E}\left(\Phi_{\theta} u, \Phi_{\sigma_{1}(1)} \sigma_{2}(1)\right) \\
& \leq \operatorname{dist}_{E}\left(\Phi_{\theta} u, \sigma_{2}(1)\right)+\operatorname{dist}_{E}\left(\sigma_{2}(1), \Phi_{\sigma_{1}(1)} \sigma_{2}(1)\right) \\
& \leq c_{\rho}^{-1} \rho+\sup \left\{\operatorname{dist}_{E}\left(w, \Phi_{\alpha} w\right) ;|\alpha| \leq \rho, w \in K_{b}\right\} .
\end{aligned}
$$

Since $K_{b}$ is compact by $(P S P)_{b}$, we have

$$
R(\rho)=c_{\rho}^{-1} \rho+\sup \left\{\operatorname{dist}_{E}\left(w, \Phi_{\alpha} w\right) ;|\alpha| \leq \rho, w \in K_{b}\right\} \rightarrow 0 \quad \text { as } \rho \rightarrow 0
$$

and (3.5a) and (3.5b) hold.

Proof of Theorem 2.1(ii). For a given neighborhood $O$ of $K_{b}$, we choose $\rho>0$ so small that $N_{R(\rho)}\left(K_{b}\right) \subset O$. By Lemma 3.2, we have

$$
\pi\left(\widetilde{N}_{\rho}\left(\widetilde{K}_{b}\right)\right) \subset N_{R(\rho)}\left(K_{b}\right) .
$$

For any $\bar{\varepsilon}>0$, by Proposition 3.1 there exists $\varepsilon \in(0, \bar{\varepsilon})$ and $\widetilde{\eta} \in C([0,1] \times M)$ with the properties stated in Proposition 3.1. Then we define

$$
\eta(t, u)=\pi(\widetilde{\eta}(t, 0, u)):[0,1] \times E \rightarrow E .
$$

Then we can see that $\eta$ has the desired properties.

As a corollary to Theorem 2.1, we have

Corollary 3.1. Suppose that Assumption 2.1 and $(P S P)_{b}$ hold. Moreover suppose $K_{b}=\varnothing$. Then there exists $\varepsilon>0$ such that $[I \leq b+\varepsilon]$ is deformable into $[I \leq b-\varepsilon]$.

Remark 3.1. From Corollary 3.1, if $(P S P)_{b}$ holds for $b \in \mathbf{R}$ and $K_{b}=\varnothing$, then even if the standard critical set $\widehat{K}_{b}=\left\{u \in E ; I(u)=b, I^{\prime}(u)=0\right\}$ is not empty, $[I \leq b+\varepsilon]$ is deformable into $[I \leq b-\varepsilon]$. Thus, critical points without Pohozaev identity $P(u)=0$ do not affect topology of level sets of $I$. 


\section{Generation of $(P S P)$ sequences at minimax level}

Under the Assumption 2.1 (but without assuming (PSP) condition), we have the following existence result for $(P S P)$ sequence at minimax levels. It can be regarded as a refinement of Ekeland's principle under Assumption 2.1.

For the sake of simplicity, we state the result for Mountain Pass Theorem due to Ambrosetti and Rabinowitz [1].

Theorem 4.1. Suppose that Assumption 2.1 holds and I( $u)$ has a mountain pass geometry. That is, for $e \in E$ with $e \neq 0$, set

$$
\begin{aligned}
& \Gamma=\{\gamma \in C([0,1], E) ; \gamma(0)=0, \gamma(1)=e\}, \\
& b=\inf _{\gamma \in \Gamma} \max _{t \in[0,1]} I(\gamma(t)),
\end{aligned}
$$

and assume

$$
b>\max \{I(0), I(e)\} .
$$

Then there exists a (PSP) sequence $\left(u_{j}\right)_{j=1}^{\infty}$ at level $b$, that is, $\left(u_{j}\right)_{j=1}^{\infty}$ satisfies (2.1a)-(2.1c).

Proof. We argue indirectly and suppose that there does not exists sequences $\left(u_{j}\right)_{j=1}^{\infty}$ with (2.1a)-(2.1c). In particular, $K_{b}=\varnothing$. Then there exists $\delta_{0}>0$ such that

$$
c_{0} \equiv \inf \left\{\left(|P(u)|^{2}+\left\|I^{\prime}(u)\right\|_{E^{*}}^{2}\right)^{1 / 2} ; I(u) \in\left[b-\delta_{0}, b+\delta_{0}\right]\right\}>0 .
$$

By (3.2a), (3.3), we have

$$
\|D J(\theta, u)\|_{(\theta, u), *} \geq c_{0}, \quad \text { if } J(\theta, u) \in\left[b-\delta_{0}, b+\delta_{0}\right] .
$$

Thus (3.4) holds. Repeating the argument in Proposition 3.1 for any $\bar{\varepsilon}>0$ with $I(0)$, $I(e)<b-\bar{\varepsilon}$ there exists $\varepsilon \in(0, \bar{\varepsilon})$ and $\widetilde{\eta} \in C([0,1] \times M, M)$ satisfying (i)-(iii) and

$$
\widetilde{\eta}\left(1,[J \leq b+\varepsilon]_{M}\right) \subset[J \leq b-\varepsilon]_{M} .
$$

Thus $\eta(t, u)=\pi(\widetilde{\eta}(t, 0, u))$ satisfies (1)-(3) and (5) in Theorem 2.1(ii). Now take a path $\gamma \in \Gamma$ with $\max _{t \in[0,1]} I(\gamma(t))<b+\varepsilon$ and consider $\widetilde{\gamma}(t)=\eta(1, \gamma(t)) \in \Gamma$. Then $\widetilde{\gamma}(t)$ satisfies $\max _{t \in[0,1]} I(\widetilde{\gamma}(t))<b-\varepsilon$, which is a contradiction.

Remark 4.1. In [16], at mountain pass level $b$ for $I(u)$, we find a (PS) sequence $\left(\theta_{j}, u_{j}\right) \subset$ $\mathbf{R} \times E$ for $J(\theta, u)$ such that as $j \rightarrow \infty$

$$
\begin{aligned}
& J\left(\theta_{j}, u_{j}\right) \rightarrow b, \\
& \partial_{u} J\left(\theta_{j}, u_{j}\right) \rightarrow 0 \text { in } E^{*}, \\
& \partial_{\theta} J\left(\theta_{j}, u_{j}\right) \rightarrow 0, \\
& \theta_{j} \rightarrow 0 .
\end{aligned}
$$

This sequence is obtained by applying Ekeland's principle to $J(\theta, u)$ in [16].

Clearly in this setting, $\left(\Phi_{\theta_{j}} u_{j}\right)$ is a $(P S P)_{b}$ sequence for $I(u)$. See also [23] for generation of $(P S P)$ sequences. 


\section{An application to nonlinear elliptic problems in strip-like domains}

Our abstract results can be applied to many problems. For example, nonlinear scalar fields equations, nonlinear Choquard equations, etc.

Here we give an application to a nonlinear elliptic problems in a strip-like domain:

$$
\begin{aligned}
-\Delta u & =f(u) \text { in } \mathbf{R}^{k} \times D, \\
u & \in H_{0}^{1}\left(\mathbf{R}^{k} \times D\right),
\end{aligned}
$$

where $k \geq 2$ and $D \subset \mathbf{R}^{\ell}(\ell \geq 1)$ is a bounded open domain with a smooth boundary $\partial D$.

In what follows we write an element in $\mathbf{R}^{k} \times D$ by $(x, y)\left(x=\left(x_{1}, \cdots, x_{k}\right) \in \mathbf{R}^{k}\right.$, $\left.y=\left(y_{1}, \cdots, y_{\ell}\right) \in \mathbf{R}^{\ell}\right)$ and $\nabla_{x}=\left(\partial_{x_{1}}, \cdots, \partial_{x_{k}}\right), \nabla_{y}=\left(\partial_{y_{1}}, \cdots, \partial_{y_{\ell}}\right), \nabla_{x, y}=\left(\nabla_{x}, \nabla_{y}\right)$.

We define

$$
I(u)=\int_{\mathbf{R}^{k} \times D}\left(\frac{1}{2}\left|\nabla_{x, y} u\right|^{2}-F(u)\right) d x d y: H_{0}^{1}\left(\mathbf{R}^{k} \times D\right) \rightarrow \mathbf{R},
$$

where $F(\xi)=\int_{0}^{\xi} f(\tau) d \tau$. Solutions of (5.1) are characterized as critical points of $I(u)$.

Defining

$$
\mathcal{G}(v)=\int_{D}-\frac{1}{2}\left|\nabla_{y} v\right|^{2}+F(v) d y: H_{0}^{1}(D) \rightarrow \mathbf{R},
$$

formally we have

$$
I(u)=\int_{\mathbf{R}^{k}} \frac{1}{2}\left\|\nabla_{x} u(x, y)\right\|_{L^{2}(D)}^{2}-\mathcal{G}(u(x, y)) d x .
$$

We set $N=k+\ell \geq 3$ and we assume

(f0) $f(\xi) \in C(\mathbf{R}, \mathbf{R})$ and $f(\xi)$ is odd.

(f1) $\lim _{\xi \rightarrow \infty} \frac{f(\xi)}{\xi^{N+2}}=0$.

(f2) $m_{0} \equiv \lim _{\xi \rightarrow 0} \frac{f(\xi)}{\xi}<\lambda_{1}(D)$, where $\lambda_{1}(D)$ is the first eigenvalue of $-\Delta$ under Dirichlet boundary condition.

(f3) There exists $\zeta_{0}(y) \in C_{0}^{\infty}(D)$ such that $\mathcal{G}\left(\zeta_{0}\right)>0$. in $x$ :

To find critical points of $I(u)$, we restrict $I(u)$ to a space of axially symmetric functions

$$
\begin{gathered}
H_{s, 0}^{1}\left(\mathbf{R}^{k} \times D\right)=\left\{u \in H_{0}^{1}\left(\mathbf{R}^{k} \times D\right) ; u(x, y)=u(|x|, y)\right. \text { is axially symmetric } \\
\text { with respect to } \left.x \in \mathbf{R}^{k}\right\} .
\end{gathered}
$$

Our main result in this section is 
Theorem 5.1. Assume (f0)-(f3). Then $I(u): H_{s, 0}^{1}\left(\mathbf{R}^{k} \times D\right) \rightarrow \mathbf{R}$ has a unbounded sequence of critical values. In particular, (5.1) has infinitely many solutions which are axially symmetric in $x$, that is $u(x, y)=u(|x|, y)$.

Remark 5.1. Introducing a suitable truncation of $f(\xi)$, the condition (f1) can be relaxed to (see $[8$, Section 2])

(f1') $\lim _{\xi \rightarrow \infty} \frac{f(\xi)}{\xi^{N+2}} \leq 0$.

Remark 5.2. (i) Under condition (f0), (f1'), (f2), (f3) but without oddness of $f(\xi)$, existence of a least energy solution is shown in [8].

(ii) We refer to Esteban [13] and Grossinho [15] for earlier works. In [13,15], they study the case where $G(s) / s^{-\theta}$ is non-decreasing in $[0, \infty)$ for some $\theta>2$.

Remark 5.3. In view of (5.2), conditions (f0)-(f3) are analogies of (g0)-(g3) for elliptic problems in strip-like domains (5.1). It seems difficult to take an approach in spirit of [5, 6]: find critical points of

$$
u \mapsto \frac{1}{2}\left\|\nabla_{x} u\right\|_{L^{2}\left(\mathbf{R}^{k} \times D\right)}^{2} ;\left\{u \in H_{0}^{1}\left(\mathbf{R}^{k} \times D\right) ; \int_{\mathbf{R}^{k} \times D}-\frac{1}{2}\left|\nabla_{y} u\right|^{2}+F(u) d x d y=1\right\} .
$$

For a proof Theorem 5.1. We set

$$
E=H_{s, 0}^{1}\left(\mathbf{R}^{k} \times D\right) \quad \text { and } \quad\|u\|_{E}=\left(\left\|\nabla_{x, y} u\right\|_{L^{2}\left(\mathbf{R}^{k} \times D\right)}^{2}+\|u\|_{L^{2}\left(\mathbf{R}^{k} \times D\right)}^{2}\right)^{1 / 2} .
$$

We note that $E=H_{s, 0}^{1}\left(\mathbf{R}^{k} \times D\right)$ is compactly embedded into $L^{q}\left(\mathbf{R}^{k} \times D\right)$ for $q \in\left(2, \frac{2 N}{N-2}\right)$. See [14].

We consider the following $C^{0}$-action $\Phi: \mathbf{R} \rightarrow L(E):$

$$
\left(\Phi_{\theta} u\right)(x, y)=u\left(x / e^{\theta}, y\right) \text {. }
$$

Then

$$
\begin{aligned}
& J(\theta, u)=I\left(\Phi_{\theta} u\right)=I\left(u\left(x / e^{\theta}, y\right)\right) \\
= & \frac{1}{2} e^{(k-2) \theta}\left\|\nabla_{x} u\right\|_{L^{2}\left(\mathbf{R}^{k} \times D\right)}^{2}+\frac{1}{2} e^{k \theta}\left\|\nabla_{y} u\right\|_{L^{2}\left(\mathbf{R}^{k} \times D\right)}^{2}-e^{k \theta} \int_{\mathbf{R}^{k} \times D} F(u) d x d y \\
= & \frac{1}{2} e^{(k-2) \theta}\left\|\nabla_{x} u\right\|_{L^{2}\left(\mathbf{R}^{k} \times D\right)}^{2}-e^{k \theta} \int_{\mathbf{R}^{k}} \mathcal{G}(u) d x \in C^{1}(\mathbf{R} \times E, \mathbf{R})
\end{aligned}
$$

and $E, \Phi_{\theta}$ satisfy the Assumption 2.1. We also define $P(u): E \rightarrow \mathbf{R}$ by setting

$$
\begin{aligned}
& P(u)=\partial_{\theta} J(0, u) \\
= & \frac{k-2}{2}\left\|\nabla_{x} u\right\|_{L^{2}\left(\mathbf{R}^{k} \times D\right)}^{2}-k\left(-\frac{1}{2}\left\|\nabla_{y} u\right\|_{L^{2}\left(\mathbf{R}^{k} \times D\right)}^{2}+\int_{\mathbf{R}^{k} \times D} F(u) d x d y\right) \\
= & \frac{k-2}{2}\left\|\nabla_{x} u\right\|_{L^{2}\left(\mathbf{R}^{k} \times D\right)}^{2}-k \int_{\mathbf{R}^{k}} \mathcal{G}(u) d x .
\end{aligned}
$$

We have the following 
Proposition 5.1. Assume (f0)-(f3). Then for any $b \in \mathbf{R}, I(u)$ satisfies $(P S P)_{b}$.

This proposition is essentially shown in [8, Sections 5-6]. In fact, in [8], it is shown that if $\left(\theta_{j}, u_{j}\right) \in \mathbf{R} \times E$ satisfies

$$
\theta_{j} \rightarrow 0, \quad J\left(\theta_{j}, u_{j}\right) \rightarrow b, \quad D J\left(\theta_{j}, u_{j}\right) \rightarrow 0 \text { strongly in }(\mathbf{R} \times E)^{*},
$$

then $\left(\theta_{j}, u_{j}\right)$ has a convergent subsequences. Consider a special case $\theta_{j} \equiv 0$. It is nothing but $(P S P)_{b}$ condition.

By Proposition 5.1 we can apply Theorem 2.1 to $I(u)$ and we have deformation flow for $I(u)$. We apply Symmetric Mountain Pass Theorem to $I(u)$.

First we note that by (f2) and (f1)

$$
I(u)=\frac{1}{2} \int_{\mathbf{R}^{k} \times D}\left(\left|\nabla_{x} u\right|^{2}+\left|\nabla_{y} u\right|^{2}-m_{0}|u|^{2}\right) d x d y+o\left(\|u\|_{E}^{2}\right) \quad \text { as }\|u\|_{E} \sim 0 .
$$

Since $m_{0}<\lambda_{1}(D)$, we have for some $r_{0}, \rho_{0}>0$

$$
I(u) \geq \rho_{0} \text { for }\|u\|_{E}=r_{0} .
$$

To find symmetric mountain pass geometry, we need the following result due to [6]. To state it, we need some notation. For $n \geq 1$, we set

$$
\pi_{n-1}=\left\{\left(\xi_{1}, \cdots, \xi_{n}\right) \in \mathbf{R}^{n} ; \sum_{i=1}^{n}\left|\xi_{i}\right|=1\right\}
$$

Berestycki and Lions [6] showed the following

Proposition 5.2. For $R>0$ there exists a continuous map $\tau_{n, R}: \pi_{n-1} \rightarrow \operatorname{Lip}([0, \infty), \mathbf{R})$ such that

(1) $\operatorname{supp} \tau_{n, R}(\xi) \subset[0, R]$ for all $\xi \in \pi_{n-1}$.

(2) $\tau_{n, R}(\xi)(r) \in[-1,1]$ for all $\xi \in \pi_{n-1}$ and $r \in[0, \infty)$.

(3) $\tau_{n, R}(-\xi)(r)=-\tau_{n, R}(\xi)(r)$ for all $\xi \in \pi_{n-1}$ and $r \in[0, \infty)$.

(4) For each $\xi \in \pi_{n-1}, \tau_{n, R}(\xi)(r) \in\{+1,-1\}$ on $[0, R]$ except in at most $n$ intervals $J_{1}, \cdots, J_{p}$ of $[0, R]$, each of these intervals has length at most one. Moreover

$$
\left|\tau_{n, R}^{\prime}(\xi)(r)\right| \leq 2 \text { for all } r \in[0, \infty) .
$$

This proposition is shown in Sections 9.2a, 9.2b in [6] (set $\zeta=1$ in the argument in [6]). We note that $\tau_{n, R}(\xi)$ is explicitly given in [6]. Regarding

$$
\pi_{n-2}=\left\{\left(\xi_{1}, \cdots, \xi_{n-1}, 0\right) ; \sum_{i=1}^{n-1}\left|\xi_{i}\right|=1\right\} \subset \pi_{n-1},
$$


we have

$$
\tau_{n, R}(\xi)=\tau_{n-1, R}(\xi) \quad \text { for all } \xi \in \pi_{n-2}
$$

We also see that

$$
\begin{aligned}
& \tau_{n, R}(\xi)(r) \text { depends on continuously on } R, \\
& \tau_{n, R}(\xi)(r) \rightarrow 0 \text { as } R \rightarrow 0^{+} \text {uniformly in }(\xi, r) .
\end{aligned}
$$

For $\xi \in \mathbf{R}^{k}$, we write

$$
|\xi|_{1}=\sum_{i=1}^{n}\left|\xi_{i}\right|
$$

and we define $\gamma_{0 n}: \mathbf{R}^{n} \rightarrow E$ by

$$
\gamma_{0 n}(\xi)(x, y)= \begin{cases}\tau_{n,|\xi|_{1}}\left(\frac{\xi}{|\xi|_{1}}\right)(|x|) \zeta_{0}(y) & \text { for } \xi \neq 0, \\ 0 & \text { for } \xi=0 .\end{cases}
$$

Here $\zeta_{0}(y)$ is given in (f3). We also note that by (5.4)

$$
\gamma_{0 n}\left(\xi_{1}, \cdots, \xi_{n-1}, 0\right)=\gamma_{0, n-1}\left(\xi_{1}, \cdots, \xi_{n-1}\right) .
$$

Lemma 5.1. For each $n \in \mathbf{N}$ there exists $R_{n}>0$ such that

$$
I\left(\gamma_{0 n}(\xi)\right)<0 \text { for } \xi \in \mathbf{R}^{n} \text { with }|\xi|_{1} \geq R_{n} .
$$

Proof. Let $u(x, y)=\gamma_{0 n}(\xi)(x, y)$ for $|\xi|_{1}=R$. We have by the definition of $\tau_{n, R}(\xi)$ and Proposition 5.2(1)-(4)

$$
\begin{aligned}
& \operatorname{supp} u(x, y) \subset B_{R} \times \bar{D}, \\
& \operatorname{meas}\left\{x \in \mathbf{R}^{k} ;|x| \leq R, u(x, y) \neq \pm \zeta_{0}(y)\right\} \subset \operatorname{meas}\left(B_{R} \backslash B_{R-n}\right) .
\end{aligned}
$$

Here we use notation: $B_{R}=\left\{x \in \mathbf{R}^{k} ;|x| \leq R\right\}$. Since

$$
\left|\nabla_{x} u(x, y)\right|=\left|\nabla_{x} \tau_{n, R}\left(\frac{\xi}{R}\right)(|x|)\right|\left|\zeta_{0}(y)\right|,
$$

so

$$
\begin{array}{ll}
\nabla_{x} u(x, y)=0, & \text { if } u(x, y)= \pm \zeta_{0}(y), \\
\left|\nabla_{x} u(x, y)\right| \leq 2\left|\zeta_{0}(y)\right|, & \text { otherwise. }
\end{array}
$$

Thus, setting $C_{1}=2 \int_{D}\left|\zeta_{0}(y)\right|^{2} d y$,

$$
\frac{1}{2} \int_{\mathbf{R}^{k} \times D}\left|\nabla_{x} u\right|^{2} d x d y \leq C_{1} \operatorname{meas}\left(B_{R} \backslash B_{R-n}\right) .
$$


On the other hand, setting $C_{2}=\max _{t \in[0,1]}\left|\mathcal{G}\left(t \zeta_{0}(y)\right)\right|$, we have

$$
\int_{\mathbf{R}^{k}} \mathcal{G}(u(x, y)) d x \geq \mathcal{G}\left(\zeta_{0}\right) \text { meas }\left(B_{R-n}\right)-C_{2} \text { meas }\left(B_{R} \backslash B_{R-n}\right) .
$$

Thus we have

$$
I(u) \leq-\mathcal{G}\left(\zeta_{0}\right) \text { meas }\left(B_{R-n}\right)+\left(C_{1}+C_{2}\right) \text { meas }\left(B_{R} \backslash B_{R-n}\right) .
$$

Since $\mathcal{G}\left(\zeta_{0}\right)>0$, we have $I(u)<0$ for large $R$. Thus for large $R_{n}>0$ we have the conclusion of Lemma 5.1.

Proof of Theorem 5.1. By Lemma 5.1, we choose $R_{1}, R_{2}, \cdots$, so that

$$
R_{1}<R_{2}<\cdots<R_{n}<R_{n+1}<\cdots,
$$

such that

$$
I\left(\gamma_{0 n}(\xi)\right)<0 \quad \text { for } \xi \in \mathbf{R}^{n} \quad \text { with }|\xi|_{1}=R_{n} .
$$

We may also assume

$$
\left\|\gamma_{0 n}(\xi)\right\|_{E} \geq r_{0} \quad \text { for } \xi \in \mathbf{R}^{n} \text { with }|\xi|_{1} \geq R_{1},
$$

where $r_{0}>0$ is given in (5.3).

Now we define a sequence of minimax values. We set for $n \in \mathbf{N}$

$$
\begin{aligned}
& D_{n}=\left\{\xi \in \mathbf{R}^{n} ;|\xi|_{1} \leq R_{n}\right\}, \\
& \Gamma_{n}=\left\{\gamma \in C\left(D_{n}, E\right) ; \gamma(\xi)=\gamma_{0 n}(\xi) \text { for } \xi \in \partial D_{n}\right\},
\end{aligned}
$$

and we define

We note that by (5.7)

$$
b_{n}=\inf _{\gamma \in \Gamma_{n}} \max _{\xi \in D_{n}} I(\gamma(\xi)) .
$$

$$
\gamma\left(D_{n}\right) \cap\left\{u \in E ;\|u\|_{E}=r_{0}\right\} \neq \varnothing .
$$

Thus, $b_{n} \geq \rho_{0}$ for each $n \in \mathbf{N}$ and we can see that $b_{n}$ is a critical value of $I(u)$ for each $n \in \mathbf{N}$. To show the multiplicity of critical points, we need another set of minimax values. Modifying the definition in [24], we set

$$
\begin{aligned}
\Lambda_{n}= & \left\{\gamma\left(\overline{D_{n+p} \backslash Y}\right) ; p \geq 0, \gamma \in \Gamma_{n+p}, Y \subset D_{n+p} \backslash\{0\}\right. \text { is closed, symmetric } \\
& \text { with respect to } 0 \text { and genus }(Y) \leq p\}, \\
c_{n}= & \inf _{A \in \Lambda_{n}} \max _{u \in A} I(u) .
\end{aligned}
$$

Here genus $(Y)$ is the genus of $Y$. Clearly we have

$$
b_{n} \geq c_{n} \text { for } n \in \mathbf{N} \text {. }
$$

By our deformation result (Theorem 5.1), we can apply the argument in [24] to $c_{n}$. In particular, since $I(u)$ satisfies $(P S P)_{b}$ for any $b \in \mathbf{R}$, we have

$$
c_{n} \rightarrow \infty \text { as } n \rightarrow \infty \text {. }
$$

Thus $I(u)$ has unbounded sequence of critical values. 


\section{Acknowledgments}

The first author is supported by PRIN 2017JPCAPN "Qualitative and quantitative aspects of nonlinear PDEs" and by INdAM-GNAMPA. The second author is supported in part by Grant-in-Aid for Scientific Research (JP19H00644, JP18KK0073, JP17H02855, JP16K13771 and JP26247014) of Japan Society for the Promotion of Science.

\section{References}

[1] A. Ambrosetti, and P. H. Rabinowitz, Dual variational methods in critical point theory and applications, J. Funct. Anal., 14 (1973), 349-381.

[2] V. Ambrosio, Mountain pass solutions for the fractional Berestycki-Lions problem, Adv. Differential Equations, 23 (2018), 455-488.

[3] A. Azzollini, P. d'Avenia, and A. Pomponio, Multiple critical points for a class of nonlinear functionals, Ann. Mat. Pura Appl., 190 (2011), 507-523.

[4] T. Bartsch, and S. de Valeriola, Normalized solutions of nonlinear Schrödinger equations, Arch. Math. (Basel), 100 (2013), 75-83.

[5] H. Berestycki, and P.-L. Lions, Nonlinear scalar field equations. I. Existence of a ground state, Arch. Rational Mech. Anal., 82 (1983), 313-345.

[6] H. Berestycki, and P.-L. Lions, Nonlinear scalar field equations. II. Existence of infinitely many solutions, Arch. Rational Mech. Anal., 82 (1983), 347-375.

[7] H. Berestycki, T. Gallouët, and O. Kavian, Équations de champs scalaires euclidiens non linéaires dans le plan, C. R. Acad. Sci. Paris Ser. I Math., 297 (1983), 307-310.

[8] J. Byeon, and K. Tanaka, Nonlinear elliptic equations in strip-like domains, Adv. Nonlinear Studies, 12 (2012), 749-765.

[9] C.-N. Chen, and K. Tanaka, A variational approach for standing waves of FitzHughNagumo type systems, J. Differential Equations, 257 (2014), 109-144.

[10] S. Cingolani, M. Gallo, and K. Tanaka, Normalized solutions for fractional nonlinear scalar field equation via Lagrangian formulation, https://arxiv.org/abs/2103.10747, arXiv:2103.10747.

[11] S. Cingolani, and K. Tanaka, Ground state solutions for the nonlinear Choquard equation with prescribed mass, INdAM Springer Series: Geometric Properties for Parabolic and Elliptic PDE's, Cortona 2019 (to appear).

[12] S. Coleman, V. Glaser, and A. Martin, Action minima among solutions to a class of Euclidean fields equations, Commun. Math. Phys., 58 (1978), 211-221.

[13] M. J. Esteban, Nonlinear elliptic problems in strip-like domains: symmetry of positive vortex rings, Nonlinear Anal., 7 (1983), 365-379.

[14] M. J. Esteban, and P.-L. Lions, A compactness lemma, Nonlinear Anal., 7 (1983), 381-385.

[15] M. R. Grossinho, Some existence and bifurcation results for nonlinear elliptic problems in strip-like domains, Ricerche Mat., 36 (1987), 127-138.

[16] J. Hirata, N. Ikoma, and K. Tanaka, Nonlinear scalar field equations in $\mathbf{R}^{N}$ : mountain pass and symmetric mountain pass approaches, Topol. Methods Nonlinear Anal., 35 (2010), 253276.

[17] J. Hirata, and K. Tanaka, Nonlinear scalar field equations with $L^{2}$ constraint: mountain pass and symmetric mountain pass approaches, Adv. Nonlinear Study, 19 (2019), 263-290. 
[18] N. Ikoma, Existence of solutions of scalar field equations with fractional operator, J. Fixed Point Theory Appl., 19 (2017), 649-690.

[19] N. Ikoma, Erratum to: Existence of solutions of scalar field equations with fractional operator, J. Fixed Point Theory Appl., 19 (2017), 1649-1652.

[20] N. Ikoma, Multiplicity of radial and nonradial solutions to equations with fractional operators, Commun. Pure Appl. Anal., 19 (2020), 3501-3530.

[21] N. Ikoma, and K. Tanaka, A note on deformation argument for $L^{2}$ normalized solutions of nonlinear Schrödinger equations and systems, Adv. Differential Equations, 24 (2019), 609646.

[22] L. Jeanjean, Existence of solutions with prescribed norm for semilinear elliptic equations, Nonlinear Anal., 28 (1997), 1633-1659.

[23] V. Moroz, and J. Van Schaftingen, Existence of groundstates for a class of nonlinear Choquard equations, Trans. Amer. Math. Soc., 367 (2015), 6557-6579.

[24] P. H. Rabinowitz, Minimax methods in critical point theory with applications to differential equations, CBMS Regional Conference Series in Mathematics, 65 (1986). 